\title{
Drawing Graphs in the Plane with a Prescribed Outer Face and Polynomial Area
}

\author{
Erin W. Chambers ${ }^{1}$, David Eppstein ${ }^{2}$, \\ Michael T. Goodrich ${ }^{2}$, and Maarten Löfller ${ }^{2}$ \\ 1 Dept. of Math and Computer Science, Saint Louis Univ., USA \\ echambe5@slu.edu \\ 2 Computer Science Dept., University of California, Irvine, USA \\ \{eppstein,goodrich, loffler\}@ics.uci.edu
}

\begin{abstract}
We study the classic graph drawing problem of drawing a planar graph using straight-line edges with a prescribed convex polygon as the outer face. Unlike previous algorithms for this problem, which may produce drawings with exponential area, our method produces drawings with polynomial area. In addition, we allow for collinear points on the boundary, provided such vertices do not create overlapping edges. Thus, we solve an open problem of Duncan et al., which, when combined with their work, implies that we can produce a planar straight-line drawing of a combinatorially-embedded genus- $g$ graph with the graph's canonical polygonal schema drawn as a convex polygonal external face.
\end{abstract}

\section{Introduction}

The study of planar graphs has been a driving force for graph theory, graph algorithms, and graph drawing. Our interest in this paper is in drawing planar graphs without edge crossings using straight line segments for edges, in such a way that all faces are convex polygons and the outer face is a given shape. Figure 1 shows an example.

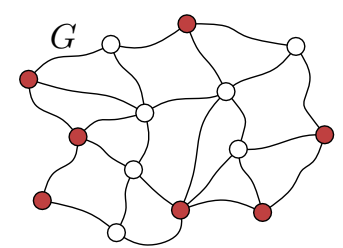

(a)

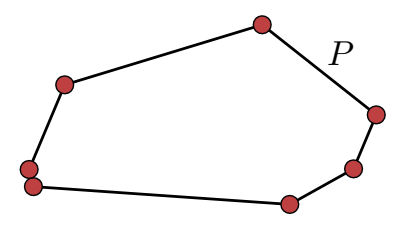

(b)

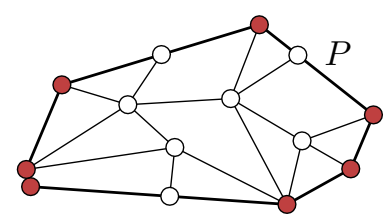

(c)

Fig. 1. Our problem: given (a) a combinatorially embedded planar graph $G$ and (b) a polygon $P$ with certain vertices on the outer face of $G$ marked as corresponding to vertices of $P$, find (c) a straight-line embedding of $G$ that uses $P$ as the shape of its outer face 
Related Prior Work. In seminal work that has been highly influential in graph drawing, Tutte [1920] shows that one can draw any planar graph using non-crossing straight-line edges so that the outer face is drawn as a prescribed convex polygon. This work has influenced a host of subsequent papers, and, according to Google Scholar, Tutte's 1963 paper has been directly cited over 600 times. His work has influenced many methods for drawing maximal planar graphs [101721] and drawing planar graphs using straight-line edges (e.g., see 345 5 11 7 14 1 16]). Moreover, not only has Tutte's result itself been highly influential, but because his method is based on a force-directed layout method, it has also influenced a considerable amount of work on force-directed layouts (e.g., see 68121318]).

Unfortunately, one drawback of Tutte's algorithm is that it can result in drawings with exponential area. This area blowup is not inherent in planar straight-line drawing, but known polynomial-area straight-line drawing algorithms (e.g., 45111416) lose a critical feature of Tutte's drawing algorithm, in that none of them allow the vertices of a planar graph's outer face to be placed on a prescribed convex polygon. Becker and Hotz [2], on the other hand, show how to draw a planar graph with minimum weighted edge length and prescribed outer face but, like Tutte's method, their method may produce drawings with exponential area. Duncan et al. 9] pose as an open problem whether an algorithm can produce polynomial-area straight-line drawings with vertices on a given convex polygon.

One motivation for prescribing the outer face of a planar drawing comes from a common way of drawing planar representations of genus- $g$ graphs. Namely, if a graph $G$ is embedded into a genus- $g$ topological surface, the surface may be cut along the edges and vertices of $2 g$ fundamental cycles in $G$ to form a topological disk (known as a canonical polygonal schema), with a boundary that is made up of $4 g$ paths (with multiple copies of the vertices on the fundamental cycles). Moreover, as shown by Duncan et al. 9, $G$ can be cut in this way so that each of these $4 g$ paths is chord-free, that is, so that there are no non-path edges between two vertices strictly internal to the same path. The standard way of drawing this unfolded version of such an embedding, in the topology literature (e.g., see [15]), is to draw the disk as a convex polygon with each of its $4 g$ boundary paths drawn as a straight line segment: the geometric shape is used to make clear the pattern in which the surface was cut to form a disk. Fortunately, given Tutte's seminal result, it is possible to draw any chord-free canonical polygonal schema in this way. The drawback of using Tutte's algorithm for this purpose is that the resulting drawing may have exponential area. Thus, we are interested in drawing the unfolded embedding in polynomial area and in polynomial time.

Our Results. In this paper, we describe an algorithm for drawing a planar graph with a prescribed outer face shape. The input consists of an embedded planar graph $G$, a partition of the outer face of the embedding into a set $\mathcal{S}$ of $k$ chord-free paths, and a $k$-sided polygon $P$; the output of our algorithm is a drawing of $G$ within $P$ with each path in $\mathcal{S}$ drawn along an edge of $P$. Given the above-mentioned prior result of Duncan et al. [9, for finding chord-free canonical 
polygonal schemas, our result implies that we can solve their open problem: any graph $G$ combinatorially embedded in a genus- $g$ surface has a polynomial-area straight-line planar drawing of a canonical polygonal schema $S$ for $G$, drawn as a $4 g$-sided convex polygon $P$ with the vertices of each path in $S$ drawn along an edge of $P$.

\section{Preliminaries}

In this paper, we show how to draw a graph with a given boundary with coordinates of polynomial magnitude. Before treating the main construction, though, we show in this section that we can equivalently state the problem in terms of the resolution of the graph. Furthermore, we recall some known results and concepts.

Resolution. Instead of drawing a graph with integer coordinates of small total size, we will make a drawing with real coordinates that stays within a fixed region (inside the input polygon) with a large resolution.

Let $G$ be a graph that is embedded in $\mathbb{R}^{2}$ with straight line segments as edges. We define the resolution of $G$ to be the shortest distance between either two vertices of the graph, or between a vertex and a non-incident edge. The diameter of $G$ is the largest distance between two vertices of the graph.

We begin by establishing a relation between resolution and size, which basically says that drawing a graph $G$ with small diameter and large resolution also results in another drawing with integer coordinates and small size. Generally it may not be possible to scale a given input polygon such that its coordinates become integers, so we need to do some rounding. We say that two drawings of $G$ are combinatorially equivalent if their topology is the same, and any collinear adjacent edges in one drawing are also collinear in the other. We say two drawings are $\varepsilon$-equivalent if the distance between the locations of each vertex of $G$ in the two drawings is at most $\varepsilon$. The following lemma is proved in the full version of our paper, which can be found online at arXiv:1009.0088.

Lemma 1. Let $G$ be a graph, and let $\Gamma$ be a drawing of $G$ without crossings, with constant resolution, and with diameter $D$. Then there exists another drawing $\Gamma^{\prime}$ of $G$ with integer coordinates and diameter $O\left(D^{2}\right)$, such that a scaled copy of $\Gamma^{\prime}$ with diameter $D$ is both combinatorially equivalent and $O(1)$-equivalent to $\Gamma$.

Note that, for a fixed input polygon with non-integer vertex coordinates, this perturbation may slightly modify its shape, since it may not be possible to find a similar copy of the polygon with integer vertex coordinates.

Now, let $Q$ be a set of points in the plane. We define the potential resolution of $Q$ to be the resolution of the complete graph on $Q$. Similarly, for a polygon $P$, we define its potential resolution to be the potential resolution of its set of vertices. Clearly, the resolution of any drawing we can achieve will depend on the potential resolution of the input polygon, because the drawing could be forced to include any edge of the complete graph. 
Next, we make an observation about the nature of the potential resolution of convex polygons.

Observation 1. If $P$ is a convex polygon, then the potential resolution of $P$ is the minimum over the vertices of $P$ of the distance between that vertex and the line through its two neighboring vertices.

Thus, for a convex polygon $P$ to allow for a drawing of polynomial area in its interior, we insist that $P$ has a polynomially-bounded aspect ratio. It cannot be arbitrarily thin and still support a polynomial-area drawing in its interior.

Alpha Cuts. We now describe a useful property of the potential resolution of a convex polygon, namely that it can be "distributed" any way we want when cutting the polygon into smaller parts. This will be made more precise later. We first make another observation about convex polygons, which is proven in the full version.

Lemma 2. Let $P$ be a convex polygon, $v$ a vertex of $P$, $e$ an edge incident to $v$, and $\alpha \in(0,1)$ a number. Let $P^{\prime}$ be a copy of $P$ where $v$ has been replaced by $v^{\prime}$ by moving $v$ along $e$ over a fraction $\alpha$ of the length of $e$. Then the potential resolution of $P^{\prime}$ is at least $1-\alpha$ times the potential resolution of $P$.

Let $P$ be a convex polygon. We will show that we can cut $P$ into two smaller polygons, "distributing" its potential resolution in any way we want.

We define an $\alpha$-cut of $P$ to be a directed line $\ell$ that splits $P$ into two smaller polygons, such that if an edge $e$ of $P$ is intersected by $\ell$, the length of the piece of $e$ to the left of $\ell$ is $\alpha$ times the length of $e$, and the piece of $e$ to the right of $\ell$ is $(1-\alpha)$ times the length of $e$. For a given convex polygon and two features of its boundary (either vertices or edges), there is a unique $\alpha$-cut that cuts the polygon through those two features in order.

Lemma 3. Suppose we are given a convex polygon $P$ of resolution $d$, two features (either vertices or edges) of $P$, and a fraction $0<\alpha<1$. Let $\ell$ be the $\alpha$-cut through the two given features that cuts $P$ into a piece $P_{l}$ to the left of $\ell$ and $a$ piece $P_{r}$ to the right of $\ell$. Then the potential resolution of $P_{l}$ is at least $\alpha$ d unless the two features are two adjacent edges that meet to the right of $\ell$. Similarly, the potential resolution of $P_{r}$ is at least $(1-\alpha) d$ unless the two features are two adjacent edges that meet to the left of $\ell$.

Proof: We will argue about the potential resolution of $P_{l}$; the argument for $P_{r}$ is symmetric. We prove this lemma by applying Lemma 2 to the new vertices of $P_{l}$. If both features where $\ell$ cuts through $P$ are vertices, then all vertices of $P_{l}$ are also vertices of $P$ and clearly the potential resolution can only become better. However, if one or both of the features are edges, then $P_{l}$ has one or two new vertices that are not part of $P$. Figure 2 shows three different cases that can occur. To solve this problem, we first alter $P$ to a different polygon $P^{\prime}$ that has the new vertices. Let $u^{\prime}$ be the place where $\ell$ enters $P$ and $u$ the closest 


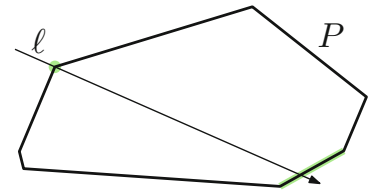

(a)

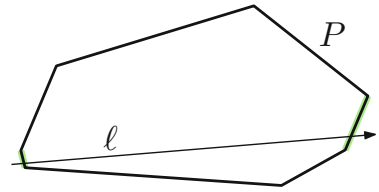

(b)

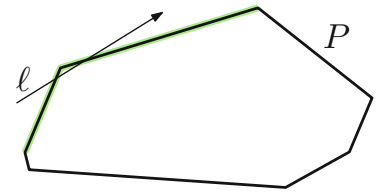

(c)

Fig. 2. (a) An $\alpha$-cut through a vertex and an edge. (b) An $\alpha$-cut through two nonadjacent edges. (c) An $\alpha$-cut through two adjacent edges.

vertex below $\ell$ along the boundary to it (possibly $u=u^{\prime}$ ), and similarly let $v^{\prime}$ be the place where $\ell$ exits $P$ and $v$ the closest vertex below $\ell$. Now, we create $P^{\prime}$ by moving $u$ to $u^{\prime}$ and $v$ to $v^{\prime}$. Clearly, both will move a fraction $1-\alpha$ along their edges, so by Lemma $2 P^{\prime}$ has a potential resolution of at most $\alpha$ times the potential resolution of $P$. Therefore, the potential resolution of $P_{l}$ can only be larger.

The only exception is when $u=v$; in this case we cannot move the vertex to two new places simultaneously, but we have to create two new vertices. Indeed, the result is not true in that case, since the two new vertices can be arbitrarily close to each other as $\alpha$ comes arbitrarily close to 1 , so the resolution of $P^{\prime}$ cannot be expressed in terms of $\alpha$, as can be seen in Figure 2 c.

Combinatorial Embeddings. Let $G=(V, E)$ be a plane graph. That is, we consider the combinatorial structure of $G$ 's embedding to be fixed, but we are free to move its vertices and edges around. Let $F$ be the set of faces of $G$, excluding the outer face. We make some definitions about faces. We say that a subset $F^{\prime} \subset F$ induces a subgraph $G\left\langle F^{\prime}\right\rangle$ of $G$ that consists of all vertices and edges that are incident to the faces in $F^{\prime}$. A subset $F^{\prime} \subset F$ is said to be vertexconnected if $G\left\langle F^{\prime}\right\rangle$ is connected; it is said to be edge-connected if the dual graph induced by the dual vertices of $F^{\prime}$ is connected. In other words, faces that share an edge are both edge-connected and vertex-connected, but faces that share only a vertex are only vertex-connected.

We recall a lemma from [9], rephrased in terms of the faces of the graph:

Lemma 4. Given an embedded plane graph $G$ that is fully triangulated except for the external face and two edges $e_{1}$ and $e_{2}$ on that external face, it is possible to partition the faces of $G$ into three sets $F_{1}, F_{2}, R \subset F$ such that:

1. All vertices of $G$ are in either $G\left\langle F_{1}\right\rangle$ or $G\left\langle F_{2}\right\rangle$.

2. $R$ is edge-connected and contains the faces incident to $e_{1}$ and $e_{2}$.

3. $F_{1}$ and $F_{2}$ are both vertex-connected.

4. The edge-connected components of $F_{1}$ and $F_{2}$ all share an edge with the outer face of $G$.

Intuitively, $R$ is a path of faces that goes from $e_{1}$ to $e_{2}$ and that splits the remaining faces into two sets $F_{1}$ and $F_{2}$. 


\section{Drawing a Graph with a Given Boundary}

We are now ready to formally state the problem and describe the algorithm to solve it.

The Problem. Let $G$ be a triangulated planar graph with a given combinatorial embedding, and let $B$ be the cycle that bounds the outer face of $G$. Let $f$ be a map from a subset of the vertices of $B$ to points in the plane, such that these points are in convex position and their order along their convex hull is the same as their order along $B$.

We say that a map $g$ from all vertices of $G$ to points in the plane respects $f$ when:

1. The vertices mapped by $f$ are also mapped by $g$ to the same points; these define a convex polygon $P$.

2. The remaining vertices of $B$ are mapped to the corresponding edges of $P$.

3. The remaining vertices of $G$ are mapped to the interior of $P$.

4. If all edges are drawn as straight line segments, they cause no crossings or incidences not present in $G$.

An example of a respectful embedding was shown in Figure 1

The input to our problem is a pair $(G, f)$. We will use the notations $B$ and $P$ as above. We will further define $F$ to be the set of faces of $G$, excluding the outer face. We define $s=|F|$, the number of internal faces, to be the size of the problem. We define $d$ to be the resolution of the problem, which is the potential resolution of $P$ (recall, that is the resolution of the complete graph on the corners of $P$ ). Our goal is to compute a mapping $g$ that respects $f$ and such that the resolution of the embedded graph is bounded by some function of $s$ and $d$.

Observe that it will not be possible to do so when there are any edges in $G$ between two vertices that have to be on the same edge of $P$. Therefore, we call a problem invalid if this is the case. We will show that for any valid problem, we can find an embedding with a polynomially bounded resolution.

The Main Idea. We want to solve the problem using divide-and-conquer. The idea is to divide $P$ into smaller convex polygons, and $F$ into smaller sets of faces, and map each subset of faces to one of the smaller regions. Then we need to decide which vertices of $G$ are mapped to the new corners of the smaller regions, and solve the subproblems.

A first idea would be to find a path in $G$ between two vertices of $B$, and lay that out on a straight line, resulting in a split of $P$ into two smaller polygons and solve the two subproblems. There are two issues with this approach though. First, the vertices on the new straight line have to be placed the same way in the two subproblems, which means they are not independent. Second, if there are any chords on this path one of the subproblems will become invalid.

To avoid these issues, we will not split along a single path, but along two paths next to each other. The region between these two paths, which we call a 


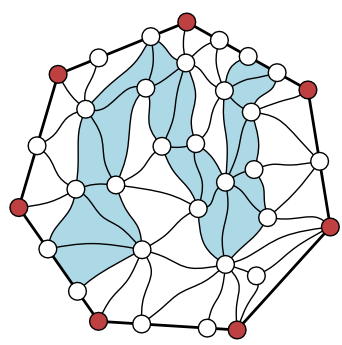

(a)

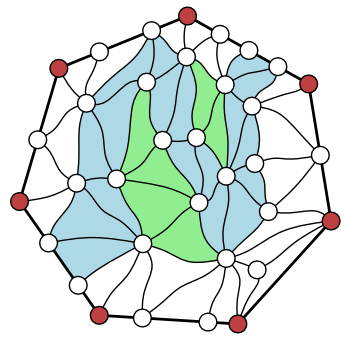

(b)

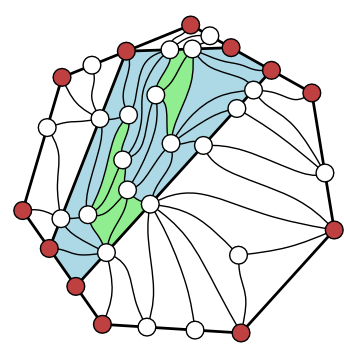

(c)

Fig. 3. (a) A "river" (a path in the dual graph that does not reuse any vertices of the primal graph) between two edges on $B$. (b) The river banks have chords, and so we include the area behind the chords in the river. (c) We fix the vertices on the river boundary that are on $B$, and draw the rest of the river boundary straight. This results in three smaller problems, plus the area of the river itself.

river, has a controlled structure, which means that we can always complete the interior independently of how the vertices on the edges were placed. Furthermore, if these paths have any chords, we shortcut them along the chords and show how to deal with the added complexity of the river. Because the river may touch the boundary of $P$ in more places, the problem may be decomposed into more than two subproblems. Figure 3(a) shows an example instance, and Figure 3(c) shows a possible decomposition where some vertices on the boundary of $P$ have been fixed, and the paths between them are made straight.

We assume the input is a valid problem with size $s$ and resolution $d$. We will keep as an invariant the ratio $d / s$, and show in the next paragraph how to subdivide a problem into smaller valid problems with the same (or better) ratio, plus an extra region (the river). We then recursively solve the independent subproblems, which results in a placement of all vertices that are not in the interior of the river. Finally, we show in the paragraph after that how to we place the vertices inside the river.

Splitting a Problem. Let $(G, P)$ be a valid problem of size $s$ and resolution $d$, and suppose that $P$ has at least four sides.

Let $e_{1}$ and $e_{2}$ be two edges of $B$ that lie on two sides of $P$ that are not consecutive. Note that the endpoints of $e_{1}$ and $e_{2}$ are not necessarily fixed yet. Now, by Lemma 4, there exists a path of faces that connects $e_{1}$ to $e_{2}$, such that the boundary of this path does not have any repeated vertices. Let $R$ be the union of the faces of $F$ on this path. We call $R$ a river; Figure 3(a) shows an example. This river may touch $B$ in other points than $e_{1}$ and $e_{2}$, so it can subdivide the faces of $F$ into any number of edge-connected subsets (apart from the river itself). We will assign a separate subproblem to each such edge-connected component.

We would like to straighten the banks of the river, but this may lead to invalid subproblems if these banks have any chords. Therefore, we identify any chords 
that the river has (note that they can only appear on the outside of the river, since the river forms a dual path), and we add the faces of $F$ behind those chords to $R$. Similarly, if one of the paths touches a side of $P$ more than once, it would create a subproblem that would be flattened. To avoid that, we also incorporate such a region into the river (even though the straight side that lies alongside $P$ is not necessarily a chord).

Next, we count the numbers of faces in the river, as well as those in the parts outside the river. Then we fix the vertices where the river touches $P$ by cutting off the subproblems, using $\alpha$-cuts where $\alpha$ is the fraction of faces inside the subproblem. Now, by Lemma 3, if we have a problem with parameters $s$ and $d$, we will construct subproblems with the same (or better) ratio $d / s$. Finally we straighten the new banks of the river, so that the subproblems have proper convex boundaries. Figure 3 shows an example.

Lemma 5. Given a valid problem $(F, P)$ where $P$ has at least four sides, We can subdivide $F$ and $P$ into disjoint sequences $F_{1}, F_{2}, \ldots, F_{h}$ and $P_{1}, P_{2}, \ldots, P_{h}$ such that each $\left(G\left\langle F_{i}\right\rangle, P_{i}\right)$ is a valid subproblem with ratio $d / s$, and such that the remainders $F^{\prime}=F \backslash \bigcup F_{i}$ and $P^{\prime}=P \backslash \bigcup P_{i}$ have the following properties:

1. $F^{\prime}$ and $P^{\prime}$ also have ratio $d / s$.

2. The vertices of $G\left\langle F^{\prime}\right\rangle$ that are not vertices of $G\left\langle\bigcup F_{i}\right\rangle$ form internally 3connected components that share at least two vertices of $G\left\langle\bigcup F_{i}\right\rangle$.

Proof: For the first part of the lemma, we need to show that the subproblems $\left(F_{i}, P_{i}\right)$ are valid and have a resolution/size ratio at least as good as $d / s$. First, we define the polygons $P_{i}$ by applying Lemma 3 to $P$ with $\alpha=s_{i} / s$ (that is, the fraction of faces in $F$ that is in $F_{i}$ ). The lemma ensures that the new polygons have potential resolution at least $d_{i} \geq \alpha d=s_{i} d / s$, so clearly $d_{i} / s_{i} \geq d / s$ as required. Second, recall that a subproblem is valid if it does not have any chords between two vertices that have to be drawn on the same side of $P_{i}$. For those sides of $P_{i}$ that are part of $P$, we already know there are no chords because $(G, P)$ was valid. For the new sides, we explicitly added all faces behind chords to the river $R=F^{\prime}$.

For the second part of the lemma, we need to show that $R$ has the right ratio and that the internal vertices of the river form 3-connected components that share two or more vertices with the boundary of the river. If we denote $s_{R}=|R|$ to be the size of the river and $d_{R}$ to be the potential resolution of the region $P^{\prime}$ in which it is to be drawn, then by Lemma 3 , the potential resolution of $R$ after repeatedly slicing off subpolygons is at least $d_{R}=d \Pi\left(1-s_{i} / s\right) \geq$ $d\left(1-\Sigma\left(s_{i} / s\right)\right)=s_{R} d / s$, so $d_{R} / s_{R} \geq d / s$. Finally, since we chose $R$ according to Lemma 4, all edge-connected pieces of $F$ outside $R$ share an edge with the outer face. In particular, this means that the boundary of $R$ does not touch itself and that any subgraphs sliced off by chords are 3-connected and share exactly two vertices with the boundary of $R$. Furthermore, if the boundary of $R$ touches the same side of $P$ multiple times, then the edge-connected components of $F$ between that side of $P$ and $R$ are also 3-connected and share at least two vertices with the new boundary of $R$. 


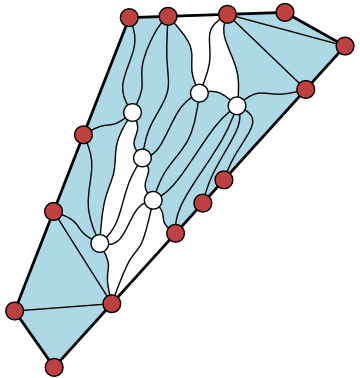

(a)

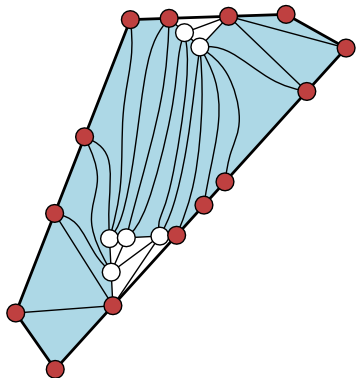

(b)

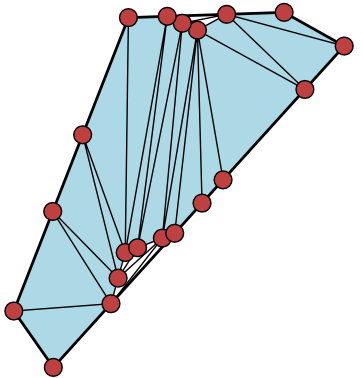

(c)

Fig. 4. (a) The interior of a river, after all vertices on its boundary have already been fixed by recursive calls to the split algorithm. (b) Because of the structure given by the river, we can identify small areas inside the river that we draw using the de FraysseixPach-Pollack algorithm. (c) If we flatten the triangular drawings enough, every vertex is able to see every other one (in fact we need slightly more, namely that no visibility ray comes too close to any vertex). Note that in the figure the drawing is not flat enough for that, but otherwise the structure would become too hard to see. In fact, in this particular case no flattening at all would be required.

When $P$ has only 3 sides, we cannot choose two edges $e_{1}$ and $e_{2}$ on non-adjacent sides of $P$. However, we can still use the same basic idea; we just have to be careful because of the special case in Lemma 3. So, let $c$ be a corner of $P$ and let $e_{1}$ and $e_{2}$ be edges of $B$ on the sides incident to $c$. The lemma does not give a bound on the resolution of the region on the far side of $c$. So, let $e_{1}$ and $e_{2}$ be the edges furthest away from $c$. Since $P$ is a triangle, the two vertices of $B$ on the far side on $e_{1}$ and $e_{2}$ are the other two corners of $P$, and they are joined by a side of $P$. This means that the region between the river and this side will be included into the river, and there will be no subproblem on the far side of $c$.

Fixing a River. It remains to show how to place the interior vertices of a river after all vertices on its boundary have been placed recursively. Again, we are given a graph $G$ and a polygon $P$ that it has to be drawn in ( $P$ is the boundary of the river, and $G$ is the part of the graph that has to be drawn in it), but there are two important differences with the initial problem: First, now we know that all vertices of $B$ have already been fixed (not only those on the corners but also those on the boundary of $P$ ). Second, we know that the remaining vertices of $G$ have a very specific structure, namely, they form internally 2-connected components that share at least two vertices with the vertices fixed along an edge. Furthermore, since all fixed vertices have been placed using the algorithm above, they will never be closer than $d / n$ to each other. This means we can draw these components using the algorithm by de Fraysseix, Pach and Pollack 11], and rotate and scale them to fit inside $P$. We can then flatten them more such that all remaining edges (between fixed vertices on the boundary of $P$ or vertices on the 
de Fraysseix-Pach-Pollack drawings) can be drawn with straight line segments. Figure 4 shows an example.

Lemma 6. Given a river placement problem, of size $s$ and resolution $d$, we can lay out the graph with a resolution of $\Omega\left(d / n^{3}\right)$.

Proof: The polygon $P$ that forms a river and the graph $G$ to be drawn in it are formed by subdividing a bigger problem according to Lemma 5 . Then, the vertices on the boundary of $P$ are placed during recursive calls to smaller problems. All vertices have to be corners of the polygons of at least one such subproblem, and by our invariant all these problems have ratio $d / s$, so the distance between any two fixed vertices cannot be smaller than $d / n$. Furthermore, Lemma 5 tells us that the vertices of $G$ can be grouped into a number of subsets $V_{1}, V_{2}, \ldots, V_{h}$ such that each $V_{i}$ is internally biconnected, and there is a sequence of at least two vertices in $V_{i}$ that is in $B$, so that have been fixed on the boundary of $P$. Note that there will be exactly two if such a component came from a chord on the river bank, but there can be more if it came from the river touching a side of $P$ multiple times. Then we can place these subgraphs using the de Fraysseix-PachPollack algorithm, starting from the vertices that are already fixed (at distance at least $d / n$ ) and adding the remaining $O\left(\left|V_{i}\right|\right)$ vertices one by one using $45^{\circ}$ edges. This results in a drawing with resolution which can be roughly bounded by $d / n^{2}$. Then, it is sufficient to squeeze them by a factor of $n$ to make sure that they do not block any potential edges, and a further factor 2 to make sure that the tips of the de Fraysseix-Pach-Pollack drawings are in fact far enough away from these potential edges, guaranteeing a good resolution. This means the final resolution of the drawing is $\Omega\left(d / n^{3}\right)$.

Putting It Together. To conclude, Lemmas 5 and 6 together imply:

Theorem 1. Given a plane graph $G$ with $n$ vertices, a convex polygon $P$ with $k$ corners and potential resolution $d$, and a map $f$ that maps $k$ vertices on the outer face of $G$ to the $k$ corners of $P$, we can draw a $G$ in $P$ respecting $f$ using resolution $\Omega\left(d / n^{3}\right)$.

Note that by Lemma 1, we can rephrase this in terms of the more standard area of a drawing when all coordinates are integer.

Corollary 1. Given a plane graph $G$ with $n$ vertices, a convex polygon $P$ with $k$ corners, at integer coordinates and diameter $D$, and a map $f$ that maps $k$ vertices on the outer face of $G$ to the $k$ corners of $P$, we can draw the graph $G$ in a scaled copy $P^{\prime}$ of $P$ that has diameter $O\left(D^{4} n^{6}\right)$, such that the drawing respects $f$ and uses only integer coordinates for the vertices of $G$.

Proof: First of all, if $P$ has only integer coordinate vertices and diameter $D$, then its potential resolution is at least $1 / D$. To see this, consider a triangle formed by any three vertices of $P$ : this triangle has area at least $1 / 2$, and in any direction its base is at most $D$ so its height must be at least $1 / D$. 
Now, by Theorem 1, we can draw $G$ inside $P$ with resolution $\Omega\left(d / n^{3}\right)=$ $\Omega\left(1 / D n^{3}\right)$. Then we can blow up the drawing by a factor $D n^{3}$, which results in a polygon of diameter $D^{2} n^{3}$ and at least constant resolution. By Lemma 1 . there now also exists a drawing of $G$ in a polygon $P^{\prime}$ of diameter $O\left(D^{4} n^{6}\right)$ in which all vertices are drawn with integer coordinates.

\section{Application to Drawing Graphs of Genus $g$}

As mentioned in the introduction, graphs of genus $g$ are often drawn in the plane by drawing their polygonal schema in a prescribed convex polygon. Using a canonical polygon schema allows us to draw this outer face as a regular $4 g$-gon that has some pairs of edges identified, and vertices on those edges duplicated. Given previous work by Duncan et al. [9, which gives us a chord-free polygonal schema derived from a graph $G$ combinatorially-embedded in a genus- $g$ surface, we can complete a straight-line drawing of $G$ using a given regular $4 g$-gon as its external face, by applying Theorem 1 and rounding the coordinates. Since a regular $4 g$-gon with diameter 1 has potential resolution $\Theta\left(1 / g^{2}\right)$, this results in a drawing with resolution $\Omega\left(1 / g^{2} n^{3}\right)$.

Of course, there is a slight issue with using a regular $4 g$-gon: not every regular $k$-gon can be embedded with fractional coordinates. So, such a drawing will not fit exactly on an integer grid no matter how big the integers can be. Thus, we either have to allow for non-integer coordinates or allow for a slight (possibly imperceptible) perturbation of the vertex coordinates.

\section{Conclusion and Open Problems}

We have given an algorithm to draw any combinatorially-embedded planar graph with a prescribed convex shape as its outer face and polynomial area, with respect to the potential resolution of that shape. That is, if the given convex shape has a polynomially-bounded aspect ratio, then we can draw the graph $G$ in its interior using polynomial area. We have not made a strenuous attempt to optimize the exponent in this area bound. So a natural open problem is to determine the upper and lower bound limits of this function.

With respect to drawings of genus- $g$ graphs using a canonical polygonal schema, although our construction guarantees that copies of corresponding vertices appearing in multiple boundary paths will be drawn in the same relative order, it does not guarantee that they will be drawn with the same inter-path distances. So another open problem is whether one can extend our algorithm to draw such paths with matching inter-path distances for corresponding vertices.

\section{Acknowledgments}

This research was supported in part by the National Science Foundation under grant 0830403, and by the Office of Naval Research under MURI grant N0001408-1-1015. 


\section{References}

1. Bárány, I., Rote, G.: Strictly convex drawings of planar graphs. Documenta Mathematica 11, 369-391, (2006) arXiv:cs/0507030 , http://www.math.uiuc.edu/documenta/vol-11/13.html

2. Becker, B., Hotz, G.: On the optimal layout of planar graphs with fixed boundary. SIAM J. Comput. 16(5), 946-972 (1987), doi:10.1137/0216061

3. Chrobak, M., Goodrich, M.T., Tamassia, R.: Convex drawings of graphs in two and three dimensions. In: Proc. 12th ACM Symp. Comput. Geom., pp. 319-328 (1996), doi:10.1145/237218.237401

4. Chrobak, M., Kant, G.: Convex grid drawings of 3-connected planar graphs. Internat. J. Comput. Geom. Appl. 7(3), 211-223 (1997), doi:10.1142/S0218195997000144

5. Chrobak, M., Payne, T.H.: A linear-time algorithm for drawing a planar graph on a grid. Inf. Proc. Lett. 54(4), 241-246 (1995), doi:10.1016/0020-0190(95)00020-D

6. Davidson, R., Harel, D.: Drawing graphs nicely using simulated annealing. ACM Trans. Graph. 15(4), 301-331 (1996), doi:10.1145/234535.234538

7. Dhandapani, R.: Greedy drawings of triangulations. Discrete Comput. Geom. 43(2), 375-392 (2010), doi:10.1007/s00454-009-9235-6

8. di Battista, G., Eades, P., Tamassia, R., Tollis, I.G.: Graph Drawing. Prentice Hall, Upper Saddle River (1999)

9. Duncan, C.A., Goodrich, M.T., Kobourov, S.G.: Planar drawings of higher-genus graphs. In: Eppstein, D., Gansner, E.R. (eds.) GD 2009. LNCS, vol. 5849, Springer, Heidelberg (2010), doi:10.1007/978-3-642-11805-0_7

10. Fáry, I.: On straight-line representation of planar graphs. Acta Sci. Math. (Szeged) 11, 229-233 (1948)

11. de Fraysseix, H., Pach, J., Pollack, R.: How to draw a planar graph on a grid. Combinatorica 10(1), 41-51 (1990), doi:10.1007/BF02122694

12. Fruchterman, T.M.J., Reingold, E.M.: Graph drawing by force-directed placement. Softw. Pract. Exp. 21(11), 1129-1164 (1991), doi:10.1002/spe.4380211102

13. Gajer, P., Goodrich, M.T., Kobourov, S.G.: A multi-dimensional approach to forcedirected layouts of large graphs. Comput. Geom. Theory Appl. 29(1), 3-18 (2004), doi:10.1016/j.comgeo.2004.03.014

14. Kant, G.: Drawing planar graphs using the canonical ordering. Algorithmica 16(1), 4-32 (1996), doi:10.1007/BF02086606

15. Lazarus, F., Pocchiola, M., Vegter, G., Verroust, A.: Computing a canonical polygonal schema of an Orientable Triangulated Surface. In: Proc. 17th ACM Symp. Comput. Geom., pp. 80-89 (2001), doi:10.1145/378583.378630

16. Schnyder, W.: Embedding planar graphs on the grid. In: Proc. 1st ACM-SIAM Symp. Discrete Algorithms, pp. 138-148 (1990),

http://portal.acm.org/citation.cfm?id=320191

17. Stein, S.K.: Convex maps. Proc. Amer. Math. Soc. 2(3), 464-466 (1951), doi:10.1090/S0002-9939-1951-0041425-5

18. Sugiyama, K., Misue, K.: Graph drawing by the magnetic spring model. J. Visual Lang. Comput. 6(3), 217-231 (1995), doi:10.1006/jvlc.1995.1013

19. Tutte, W.T.: Convex representations of graphs. Proc. London Math. Soc. 10(38), 304-320 (1960), doi:10.1112/plms/s3-10.1.304

20. Tutte, W.T.: How to draw a graph. Proc. London Math. Soc. 13(52), 743-768 (1963), doi:10.1112/plms/s3-13.1.743

21. Wagner, K.: Bemerkungen zum Vierfarbenproblem. Jber. Deutsch. Math.Verein. 46, 26-32 (1936) 